This paper has been submitted for publication to American Institute of Mathematical Sciences journal (AIMS)

Title of the paper.

\title{
Fundamental Equation of Triangles.
}

\section{Jayaram A $S^{*}$}

1 Associate professor, Department of Mechanical Engineering, Dr Ambedkar Institute Of Technology, Bangalore, ZIP 560040, Karnataka State, INDIA.

2 Affiliation : VTU, Karnataka.

* Correspondence: surya.jayaram@gmail.com; Tel: +91-897-186-2089;

Note: Some notations in equations may not display correctly in some devises.

Abstract: This paper establishes a fundamental equation $a^{n}+b^{n}=c^{n}$, applicable for any triangle, having $\mathrm{a}, \mathrm{b}$ and $\mathrm{c}$ as the sides with ' $\mathrm{c}$ ' being the longest side and $\mathrm{n}$ is a number varying from 1 to infinity. Here, $\mathrm{a}, \mathrm{b}, \mathrm{c}$ and $\mathrm{n}$ need not always be integers. It also arrives at a relation between largest angle $\mathrm{C}$ (opposite to the longest side 'c') and sides of the triangle with the equation given by, $=\cos C=\frac{\left(1+r^{2}\right)-\left\{\left(1+r^{n}\right)\right\}^{2 / n}}{2 r}$, where $\mathrm{r}=\mathrm{b} / \mathrm{a}$. The paper graphically and mathematically illustrates the relation between the angle $C$ and ' $n$ ', for different values of ' $n$ ' and ' $r$ ' both varying from 1 to infinity. The paper also shows that Pythagoras theorem is a particular case of the above fundamental equation, when $n=2$. The paper shows that the above fundamental equation is valid even when $a, b$ and $c$ are integers for non-integer values of $n>2$. This gives a new way of looking at Fermat's Last Theorem.

Keywords: (Geometry, Triangle, Equation, largest angle, ratio of sides.)

\section{Introduction}

Let us consider Pythagoras theorem. It is about relation between sides of a right angled triangle

Figure- 1 shows a right angled triangle in which $a=3$ units, $b=4$ units and $c=5$ units. According to Pythagoras theorem, we have 


$$
a^{2}+b^{2}=c^{2}
$$

Substituting the values,

$3^{2}+4^{2}=5^{2}$

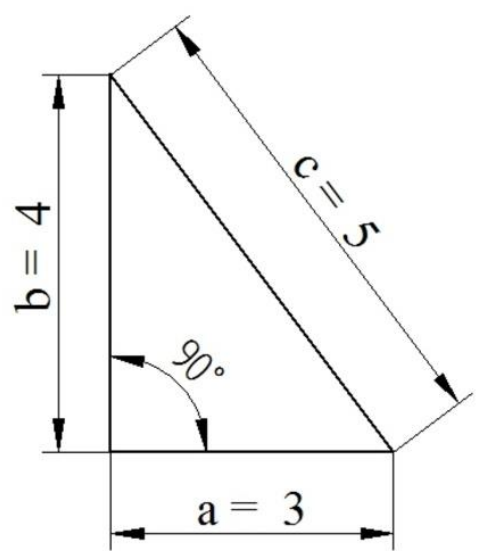

Figure 1. Right angled triangle, illustrating Pythagoras theorem.

In Pythagoras theorem, the numbers are raised to the power $n=2$.

\section{Deriving fundamental equation}

2.1. Applying cosine rule for right angled triangle.

The basic relation in any triangle with sides a, b, c and angles opposite to the sides are A,B and $\mathrm{C}$ respectively (Figure-2) are given by the cosine rule

$$
\cos C=\frac{a^{2}+b^{2}-c^{2}}{2 a b}
$$

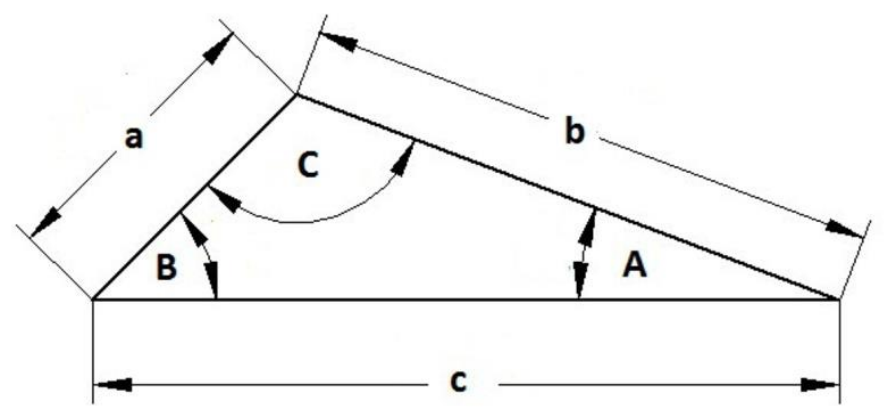

Figure 2. Triangle showing sides and angles for cosine rule. 
Let ' $a$ ' be the smallest side, and ' $c$ ' be the longest side in all the cases considered and hence the largest angle will be angle $\mathrm{C}$.

Putting $a^{2}+b^{2}=c^{2}$, in equation (2), we get

$$
\cos C=\frac{a^{2}+b^{2}-c^{2}}{2 a b}=\frac{a^{2}+b^{2}-a^{2}-b^{2}}{2 a b}=0 .
$$

So, in any triangle satisfying equation (1), the largest angle will be $\cos ^{-1} 0=90^{\circ}$ and it is the case of Pythagoras theorem.

\subsection{Putting $\boldsymbol{n}-1$}

By substituting $\mathrm{n}=1$ in equation (1), it becomes

$$
\begin{gathered}
a+b=c, \\
\text { Or } c^{2}=(a+b)^{2}
\end{gathered}
$$

Substituting this in the equation (2), we get

$$
\cos C=\frac{a^{2}+b^{2}-(a+b)^{2}}{2 a b}
$$

Expanding

the

terms

$\cos C=\frac{a^{2}+b^{2}-a^{2}-b^{2}-2 a b}{2 a b}=-1$

Hence the largest angle will be $=\cos ^{-1}(-1)=180^{\circ}$

Example: let $a=3, b=4$ units. Then, it is represented by a triangle PQR, which is a straight line of length $3+4=7$ units, with angle at $Q=180^{\circ}$ and other two angles $=0^{\circ}$ each, shown in figure 3 .

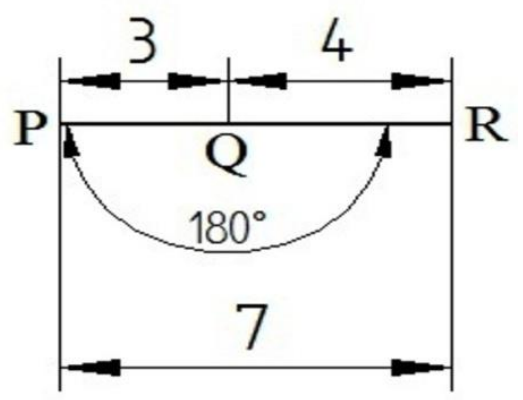

Figure 3. Figure showing triangle becoming a straight line $P Q R$. 


\subsection{Putting $\boldsymbol{n}=3$.}

When $\mathrm{n}=3$, equation (1) becomes

$$
a^{3}+b^{3}=c^{3}
$$

Note: Fermat's last theorem says all the three $a, b, c$ cannot be integers for integers of $n>2$. But in our case, they not necessarily be integers, as they are representing the sides of a triangle. Here, $n$ also need not be an integer always.

As ' $a$ ' is assumed as smallest of the three numbers, Let the ratio of b/a as 'r.'

Substituting this in equation (4), we get $a^{3}+(a r)^{3}=c^{3}$ or,

$$
a^{3}\left(1+r^{3}\right)=c^{3}
$$

Therefore, $c=\left\{a^{3}\left(1+r^{3}\right)\right\}^{1 / 3}$

On simplifying, $c=\mathrm{a}\left\{\left(1+r^{3}\right)\right\}^{1 / 3}$

So, $\left.c^{2}=a^{2}\left(1+r^{3}\right)\right\}^{2 / 3}$

Substituting this in equation (2),

$$
=\cos C=\frac{a^{2}\left(1+r^{2}\right)-a^{2}\left\{\left(1+r^{3}\right)\right\}^{2 / 3}}{2 a^{2} r}
$$

Dividing numerator and denominator by $a^{2}$, the equation reduces to

$$
=\cos C=\frac{\left(1+r^{2}\right)-\left\{\left(1+r^{3}\right)\right\}^{2 / 3}}{2 r}
$$

\section{For $\mathrm{r}=1$, angle $C=78.09^{0}$}

So, at 78.09 degrees, we get $a^{3}+b^{3}=c^{3}$

Example: The figure 4 shows a triangle $\mathrm{a}=\mathrm{b}=2$ as sides for which the closing side is 2.52 .

Substituting the values, $2^{3}+2^{3}=8+8=16=(2.52)^{3}$

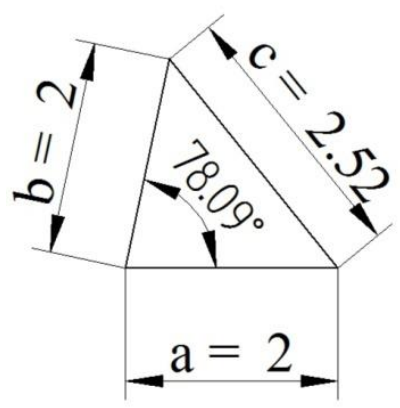


Figure 4. Triangle showing sides and angle for $n=3, r=1$.

2.4. Generalizing for the equation for ' $n$ '.

Generalizing the equation for $\mathrm{n}$, we get

$$
=\cos C=\frac{\left(1+r^{2}\right)-\left\{\left(1+r^{n}\right)\right\}^{2 / n}}{2 r}
$$

This is the general equation, giving the largest angle for $\mathbf{n}$ varying from 1 to infinity.

\section{Results}

In the equation (5), there are two variables. They are ' $r$ ' and ' $n$ '. As the side ' $a$ ' is smallest and $r=b / a$, the minimum value of ' $r$ ' is 1 because for $r<1, b$ will become smaller than ' $a$ '. The maximum value of ' $r$ ' is infinity. Similarly, the minimum value of $n=1$ and the maximum value is infinity.

Table1. Relation between characteristic angle and n. $(\mathbf{r}=\mathbf{1})$.

\begin{tabular}{|r|r|r|}
\hline $\begin{array}{r}\text { Serial } \\
\text { number }\end{array}$ & $\begin{array}{l}\text { Angle C } \\
\text { (in } \\
\text { degrees. })\end{array}$ & $\mathrm{n}$ \\
\hline 1 & 180 & 1 \\
\hline 2 & 90 & 2 \\
\hline 3 & 78.09442 & 3 \\
\hline 4 & 72.96875 & 4 \\
\hline 5 & 70.10813 & 5 \\
\hline 6 & 68.28195 & 6 \\
\hline 7 & 67.01481 & 7 \\
\hline 8 & 66.08403 & 8 \\
\hline 9 & 65.37134 & 9 \\
\hline 10 & 64.80813 & 10 \\
\hline 11 & 64.35183 & 11 \\
\hline 12 & 63.97464 & 12 \\
\hline 13 & 63.65761 & 13 \\
\hline 14 & 63.38743 & 14 \\
\hline 15 & 63.15442 & 15 \\
\hline 16 & 62.9514 & 16 \\
\hline 17 & 62.77293 & 17 \\
\hline 18 & 62.61482 & 18 \\
\hline 19 & 62.47377 & 19 \\
\hline 20 & 62.34715 & 20 \\
\hline & & \\
\hline
\end{tabular}




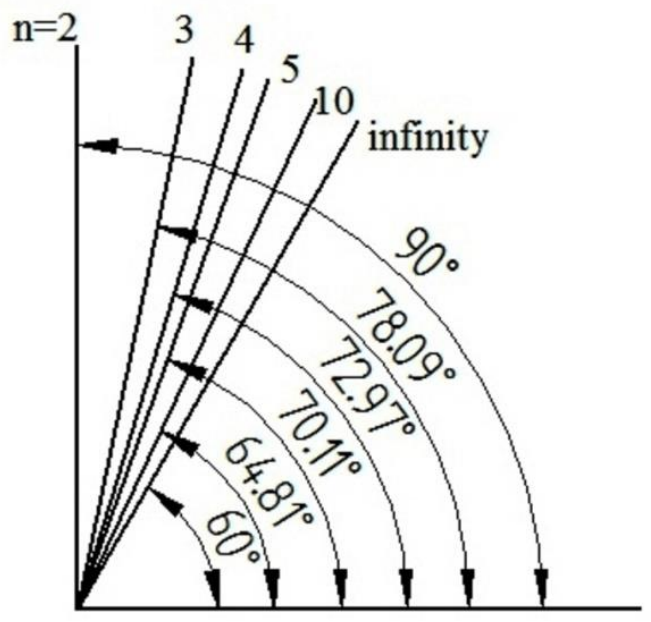

Figure 5. Pictorial view of largest angles for some values of $n$.

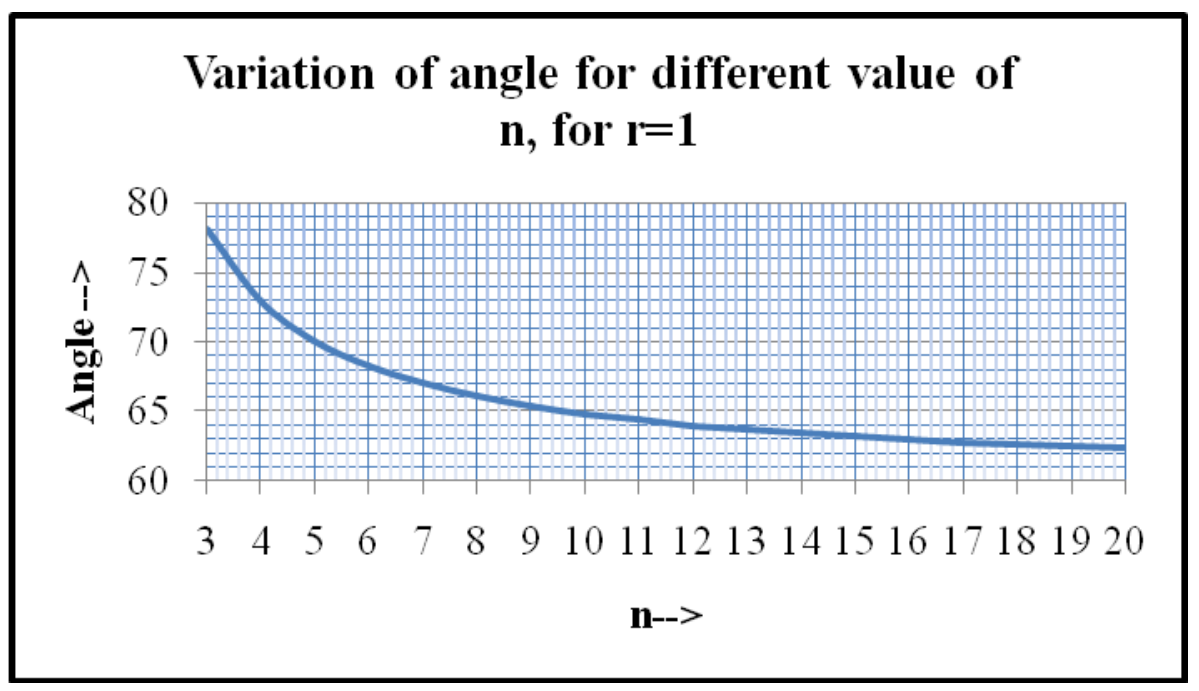

Figure 6. Relation between characteristic angle and $\mathrm{n}$ for $\mathrm{r}=1$ Above graph is drawn from $n=3$, as $n=1$ and 2 angles are same for all values of $r$

Table2 .Variation of largest angle $\mathrm{C}$ for $\mathrm{r}=2,3$ and 4 , for $\mathrm{n}=1$ to 20 .

\begin{tabular}{|r|r|r|r|}
\hline $\mathbf{n}$ & Angle C for $\mathrm{r}=2$ & Angle C for $\mathrm{r}=3$ & Angle C for $\mathrm{r}=4$ \\
\hline 1 & 180 & 180.000000 & 180.000000 \\
\hline 2 & 90 & 90.000000 & 90.000000 \\
\hline 3 & 80.31026 & 82.53881158 & 84.0177393 \\
\hline 4 & 77.33657 & 80.9419074 & 83.04455015 \\
\hline 5 & 76.25417 & 80.54920283 & 82.86434506 \\
\hline 6 & 75.82889 & 80.44576627 & 82.82864266 \\
\hline 7 & 75.65417 & 80.41731695 & 82.82125838 \\
\hline 8 & 75.58018 & 80.40925291 & 82.8196849 \\
\hline
\end{tabular}




\begin{tabular}{|r|r|r|r|}
9 & 75.54815 & 80.40691592 & 82.81934222 \\
\hline 10 & 75.53404 & 80.40622708 & 82.81926634 \\
\hline 11 & 75.52774 & 80.40602133 & 82.81924932 \\
\hline 12 & 75.5249 & 80.4059592 & 82.81924546 \\
\hline 13 & 75.5236 & 80.40594028 & 82.81924458 \\
\hline 14 & 75.523 & 80.40593447 & 82.81924437 \\
\hline 15 & 75.52273 & 80.40593267 & 82.81924433 \\
\hline 16 & 75.5226 & 80.40593212 & 82.81924432 \\
\hline 17 & 75.52254 & 80.40593194 & 82.81924431 \\
\hline 18 & 75.52251 & 80.40593189 & 82.81924431 \\
\hline 19 & 75.5225 & 80.40593187 & 82.81924431 \\
\hline 20 & 75.52249 & 80.40593187 & 82.81924431 \\
\hline
\end{tabular}

Angle C varies slightly differently for $\mathrm{r}=2,3$, and 4 for $\mathrm{n}$ varying from 3 to 20 .

\section{Angles for various values of $\mathbf{n}$ for $r=2,3$ and 4 .}

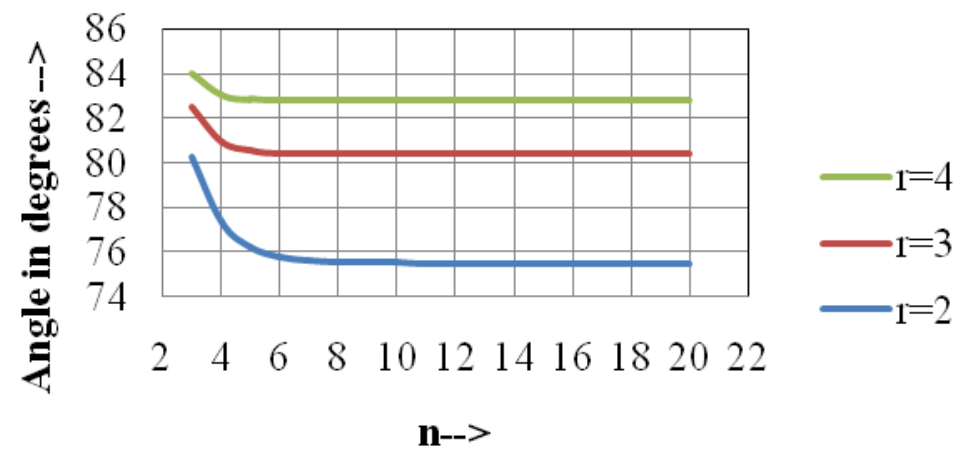

Figure -7. Variation of angle for various values of $n$ for $r=2,3$ and 4 .

We have been considering $n=1,2,3 \ldots$, but ' $n$ ' need not be an integer.

Let us consider some values of ' $n$ ' between 1 and 2 .

Table3 . Largest angles for different values of ' $n$ ' between 1 and 2.

\begin{tabular}{|r|r|r|}
\hline $\begin{array}{l}\text { Serial } \\
\text { number }\end{array}$ & $\mathrm{n}$ & $\begin{array}{l}\text { Angle } \\
\mathrm{C} \text { (degrees) }\end{array}$ \\
\hline 1 & 1 & 180.000000 \\
\hline 2 & 1.05 & 150.719466 \\
\hline 3 & 1.1 & 139.74557 \\
\hline 4 & 1.15 & 132.002996 \\
\hline 5 & 1.2 & 125.972795 \\
\hline 6 & 1.25 & 121.045363 \\
\hline 7 & 1.3 & 116.899239 \\
\hline 8 & 1.35 & 113.339379 \\
\hline 9 & 1.4 & 110.236757 \\
\hline 10 & 1.45 & 107.500848 \\
\hline 11 & 1.5 & 105.065378 \\
\hline 12 & 1.55 & 102.880226 \\
\hline
\end{tabular}




\begin{tabular}{|r|r|r|}
\hline 13 & 1.6 & 100.906516 \\
\hline 14 & 1.65 & 99.1134659 \\
\hline 15 & 1.7 & 97.4762832 \\
\hline 16 & 1.75 & 95.9747122 \\
\hline 17 & 1.8 & 94.5919996 \\
\hline 18 & 1.85 & 93.3141405 \\
\hline 19 & 1.9 & 92.1293175 \\
\hline 20 & 1.95 & 91.0274765 \\
\hline 21 & 2 & 90.0000001 \\
\hline
\end{tabular}

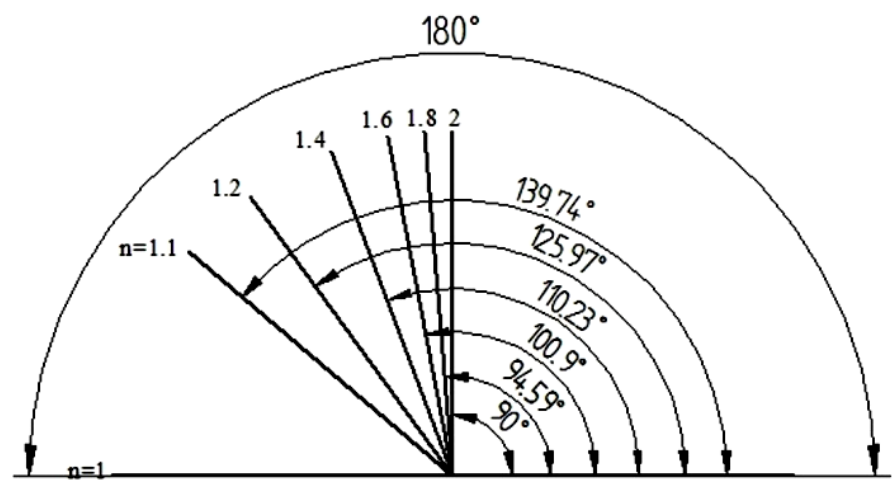

Figure 8. Pictorial view of largest angle for some values of $\mathrm{n}$ between 1 and 2 .

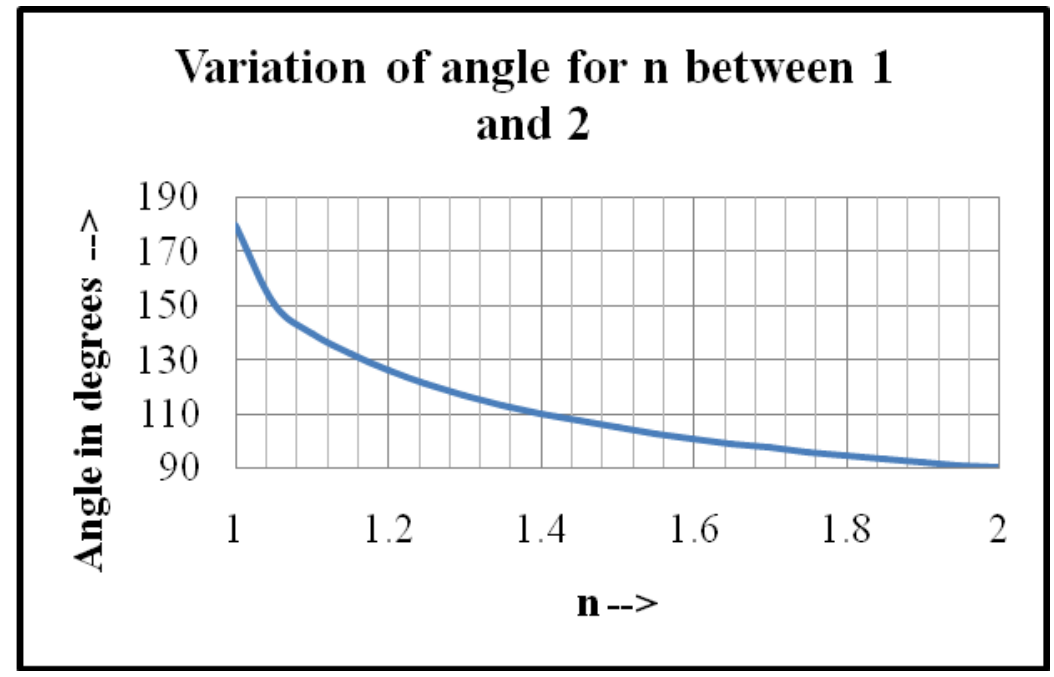

Figure 9. Variation of largest angle for values of $\mathrm{n}$ between 1 and 2 . 


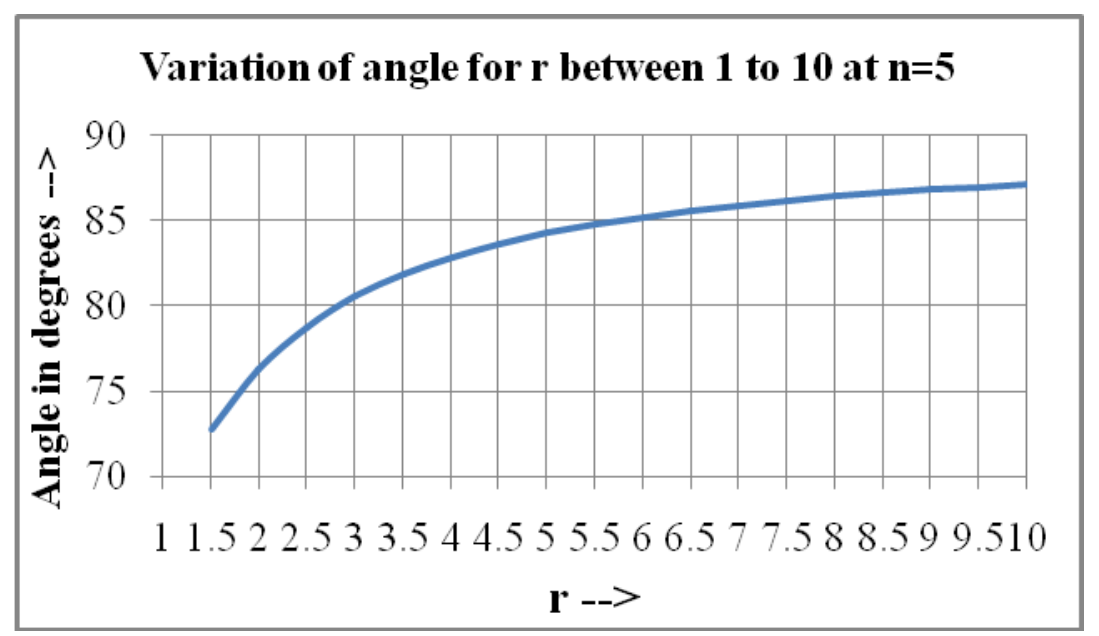

Figure 10. Variation of largest angle for values of $r$ between 1 and 10 , for $n=5$.

\section{Discussion}

4.1. It is evident that any triangle with sides $\mathrm{a}, \mathrm{b}, \mathrm{c}$ can be expressed as $a^{n}+b^{n}=c^{n}$ for which the characteristic angle $\mathrm{C}$ is given by the equation.

$=\cos C=\frac{\left(1+r^{2}\right)-\left\{\left(1+r^{n}\right)\right\}^{2 / n}}{2 r}$, where $\mathrm{r}=\mathrm{b} / \mathrm{a}$.

Pythagoras theorem is a particular case of the above equation when $\mathrm{n}=2$.

4.2. Conversely, we can find a number ' $n$ ' for any given triangle with side $a, b$ and $c$ satisfying the equation $a^{n}+b^{n}=c^{n}$.

Example. Finding 'n' for a given triangle.

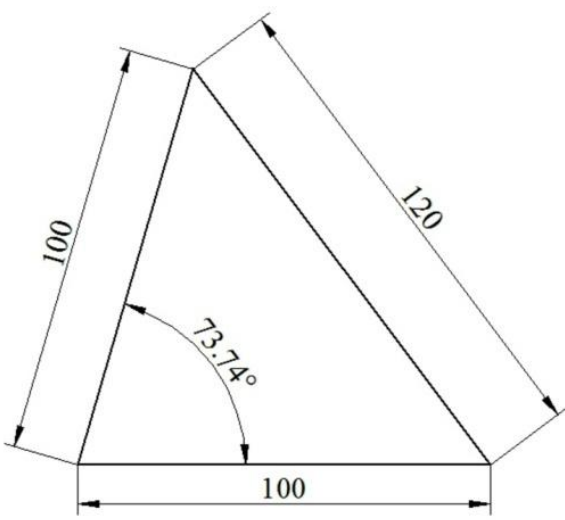

Consider the above triangle of $a=b=100$ and $c=120$. Since $a=b, r=1$.

From equation (2), we have

$=\cos C=\frac{\left(1+r^{2}\right)-\left\{\left(1+r^{n}\right)\right\}^{2 / n}}{2 r}$,

When $r=1$, the equation reduces to 
$=\cos C=\frac{2-2^{2 / n}}{2}$,

$=2 \cos C-2=-2^{\left(\frac{2}{n}\right)}$,

From cosine rule, $\quad \cos C=\frac{a^{2}+b^{2}-c^{2}}{2 a b}$

Substituting for $\mathrm{a}, \mathrm{b}, \mathrm{c}$, we get $\quad \cos C=\frac{100^{2}+100^{2}-120^{2}}{2 * 100 * 100}$

$\mathrm{C}=\cos ^{-1} 0.28$. So, angle $=73.74^{0}$

(The angle can also be directly measured and substituted in the equation, if it is possible to measure it with sufficient accuracy)

Substituting $\cos \mathrm{C}=0.28$ in equation (6), we get

$=2 * 0.28-2=-2^{\left(\frac{2}{n}\right)}$

So, $-1.44=-2^{\left(\frac{2}{n}\right)}$

Or $(1.44)^{n / 2}=2$

$n=2\left(\frac{\log 2}{\log 1.44}\right)$

Solving, we get $n=3.802$.(approx)

This is an example where sides $a, b$, and c are integers where as ' $n$ ' is not an integer but it is $>2$.

4.3. As $\mathrm{r} \rightarrow \infty$, angle $\mathrm{C}$ tends to $90^{\circ}$

From equation(2), we have

$=\cos C=\frac{\left(1+r^{2}\right)-\left\{\left(1+r^{n}\right)\right\}^{2 / n}}{2 r}$,

In the equation above, for large value of $\mathbf{r}$,

$1+r^{2} \rightarrow r^{2}$ and $\left\{\left(1+r^{n}\right)\right\}^{2 / n} \rightarrow r^{2}$

Hence the equation simplifies to 
$=\cos C=\frac{r^{2}-r^{2}}{2 r}=0 . \quad$ So, angle $\mathrm{C} \rightarrow 90^{\circ}$

This is indicated in the figure 10 .

4.4. As $n \rightarrow \infty$, largest angle $\rightarrow 60^{\circ}$ for $r=1$.

When $\mathrm{r}=1$, equation (2),

$=\cos C=\frac{2-2^{2 / n}}{2}$,

For large values of $\mathrm{n},=\cos C=\frac{2-2^{2 / n}}{2}=\frac{2-1}{2}=1 / 2$

$\cos ^{-1} 1 / 2=60^{0}$

This is indicated in the figure 6 .

4.5. Only when $n=1$ and $n=2$, all terms in cosine rule cancels out to give angle $\mathrm{C}$ as $180^{\circ}$ and $90^{\circ}$ respectively. Hence the largest angle is independent of side ratios. But for all other values of $\mathrm{n}$, angle $\mathrm{C}$ depends upon both $\mathrm{r}$ and $\mathrm{n}$.

\section{Conclusion}

5.1. Fundamental equation of any triangle with sides $\mathrm{a}, \mathrm{b}, \mathrm{c}$ is $a^{n}+b^{n}=c^{n}$, where $\mathrm{n}$ is any number between 1 to infinity.

5.2. Pythagoras theorem is a particular case of the above equation for $n=2$.

5.3. The largest angle $\mathrm{C}$ opposite to the longest side $\mathrm{c}$ can be found out by the general equation $=\cos C=\frac{\left(1+r^{2}\right)-\left\{\left(1+r^{n}\right)\right\}^{2 / n}}{2 r}$, where $\mathrm{r}=\mathrm{b} / \mathrm{a}$.

5.4. When ' $n$ ' is not an integer, $a, b$ and $c$ can be integers even for $n>2$. This has been illustrated in 4.4.2 of this paper, providing a new perspective of looking at Fermat's last theorem.

5.5. The fundamental equation of the triangle is very likely to become a stepping stone in finding a simpler proof for Fermat's last theorem.

\section{Acknowledgments}

As there are no expenses, funding was not required.

\section{Conflict of interest}

Author declares no conflict of interest.

\section{References}

1. Pythagoras- Wikipedia. https://en.wikipedia.org/wiki/Pythagoras 
2. Fermat's last theorem https://en.wikipedia.org/wiki/Fermat\%27s_Last Theorem

3. Cosine rule https://en.wikipedia.org/wiki/Law_of_cosines

4. Properties of triangles https://en.wikipedia.org/wiki/Triangle 